\title{
Force Templates - A Blueprint for Coalition Interaction within an Infosphere
}

\author{
Robert E. Marmelstein, Ph.D. \\ Air Force Research Laboratory (AFRL) - Information Directorate \\ Information Systems Division (AFRL/IFS) \\ 525 Brooks Road \\ Rome, NY 13441, USA \\ Robert.Marmelstein@rl.af.mil
}

\begin{abstract}
In this paper, we present the emerging force template model for the Joint Battlespace Infosphere (JBI) and discuss how it supports successful coalition operations. Infosphere architectures, such as the JBI, represent the way ahead for leveraging web and e-commerce technologies to streamline command, control, and intelligence (C2I) operations. We introduce the force template concept as the principal mechanism to quickly integrate battlespace entities (and their clients) into the JBI. Additionally, we show how force templates can ensure proper information dissemination within the JBI. With its emphasis on resource exchange and control, force templates provide the flexibility needed to seamlessly share information among members of ad-hoc coalitions.
\end{abstract}

\section{Introduction.}

There are many areas where technology has not caught up to military strategy and doctrine--coalition warfare is one of these. Future military operations will require close coordination and information sharing among heterogeneous units, coalition forces, and other civil and non-governmental (NGO) organizations. While United States increasingly relies on coalitions to achieve its military objectives, the technological infrastructure necessary to support this strategy has been lacking. The gulf between the desired and the possible is especially glaring in the area of C2I. For example, in the Joint Force Expeditionary Experiment (JEFX) '99, the effort to integrate coalition members into the Combined Air Operations Center (CAOC) was deemed a failure. This result was due to three factors: US-only applications within Theater Battle Management Core Systems (TBMCS), use of SIPRNET as the CAOC backbone, and the population of CAOC databases with US-only information [3]. The changes required to remedy this situation were sufficiently difficult as to result in the cancellation of the planned coalition operations in JEFX '00 [4].

One of the key recommendations from JEFX ' 99 was to develop a CAOC backbone accessible by all coalition users [3]. While some approaches include explicitly tagging database elements for releasibility, a cleaner solution requires a new paradigm that manages information in terms of standardized, discrete objects. Such an approach would enable the following positive developments:

- The segregation of information objects from their source applications and databases.

- Making publish, subscribe, query, and transformation capabilities available to producers and consumers of these information objects.

- The specification of policy governing how the published object types can be disseminated within the infosphere.

Currently, information potentially releasable to coalition partners is often combined with other, sensitive data within client applications and databases. The unfortunate result is a denial of useful information to coalition partners since the aggregated data is at a system high level. Segregating information into packages that are small, coherent, and discrete makes it easier to control and, therefore, distribute to other coalition members.

It is also possible to convert some sensitive data into a releasable form. In many cases, lightweight programs (referred to as fuselets) could be employed to accomplish these transformations. Policy associated with information objects (nominally defined by the publishers) will determine to whom, and in what form, specific objects would be disseminated. The combination of an infosphere, better information packaging, and fuselets would facilitate the controlled, secure sharing of information within a coalition. 


\section{The Joint Battlespace Infosphere.}

The JBI is a system of systems that integrates, aggregates, and distributes information to users at all echelons, from the command center to the battlefield. Infospheres are a critical stepping stone to solving the problems of coalition C2I integration because they inherently provides many of the capabilities described in the previous section. The conceptual framework for JBI was outlined in two consecutive Air Force Scientific Advisory Board (SAB) reports, Information Management to Support the Warrior (1998) [5] and Building the Joint Battlespace Infosphere (1999) [6]. The SAB vision for the JBI encompasses the four key concepts described below and in Figure 1 .

Information exchange through publish, subscribe, and query. This capability enables the user to locate and subscribe to information resources available within the JBI. Each publisher is responsible for tracking users that have subscribed to its resources. When an information resource is published, a tailored version of that resource is forwarded to the subscriber.

Transforming data to knowledge via fuselets. Fuselets are lightweight programs or scripts that process incoming information objects received from established subscriptions. When these objects arrive, fuselets can then aggregate, correlate, and/or transform them into information of interest to a given subscriber.

Distributed collaboration through shared, updateable knowledge objects. This concept refers to the ability of the JBI to facilitate collaborative problem solving among multiple, diverse users.

Assigned unit incorporation via force templates. A force template is an electronic description of an entity that enables its integration into the JBI (including all its subcomponents).

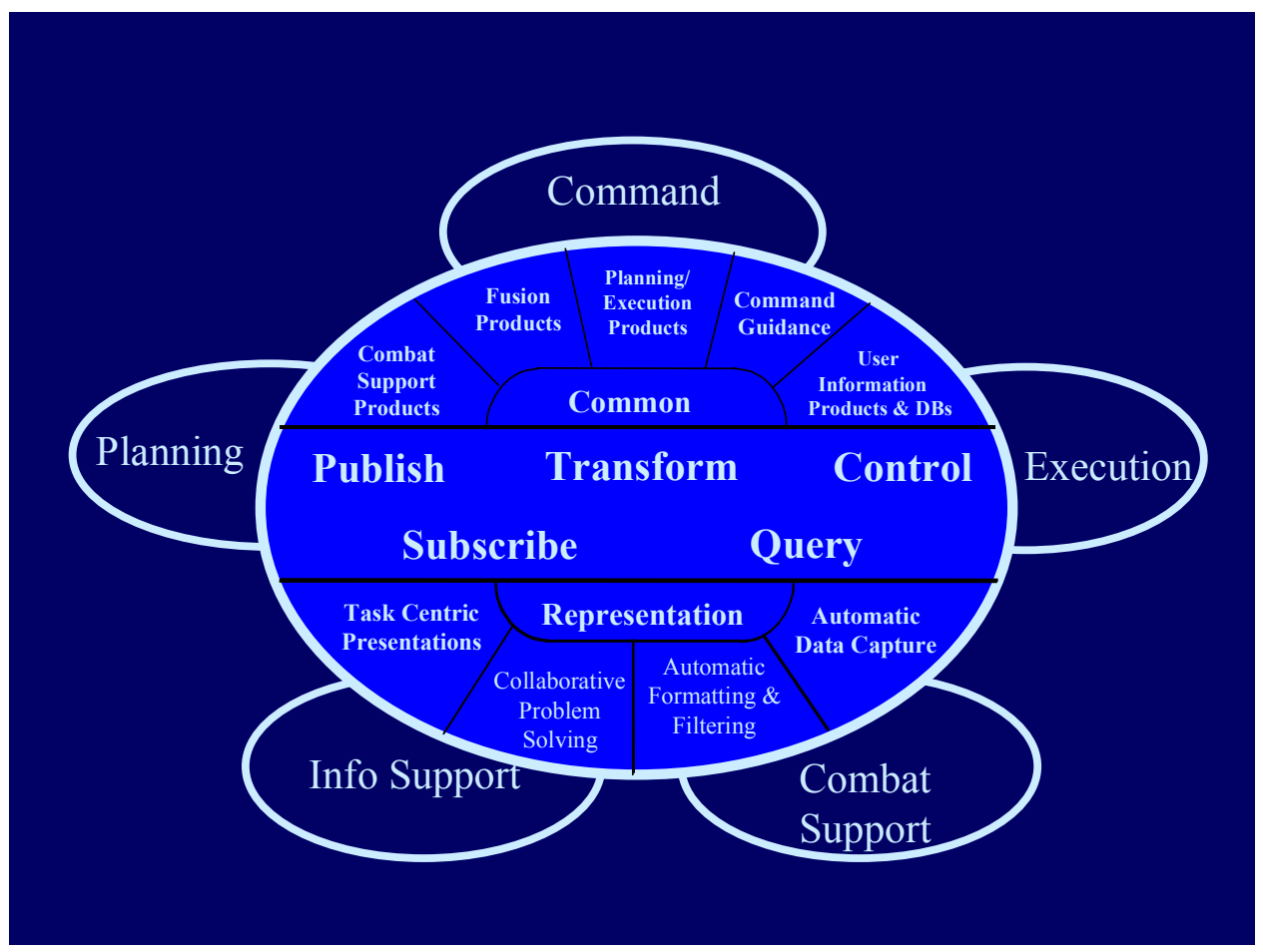

Figure 1 - JBI Capabilities 


\section{Force Template Concepts}

In this section, we build on the definition given in the last section by discussing why force templates are needed, how they model coalition units, and what information they provide to the JBI.

Why are force templates needed? While the JBI provides platform for information transfer, others must provide the content. For an infosphere to have value, the participating entities must "plug in" and use it to exchange information and service resources. The force template contains the information that enables operational entities within the battlespace (and their clients) to quickly interact using the JBI platform.

The force template also includes the context and policy that define an entity's contract with the JBI. One of the key motivations for developing the force template concept is the need to allow the JBI to grow (shrink) in a modular fashion that reflects the phase of the associated military operation. In short, the JBI must handle dramatic and sudden content changes while maintaining an acceptable level of service. Without the force template mechanism, it becomes extremely difficult to track and manage the changes to JBI content resulting from the arrival and departure of coalition units.

Entities, Clients, and Passes. An entity is an organization that decomposes into multiple components. Those components may either be other entities (child entities) or clients. In this model, entities primarily correspond to operational military units and the organizations that support them. Both parent and child entities may have their own force templates. For example, a wing and its associated squadrons may each have their own force templates. These templates may be separate, but linked based on their relationship. The level at which force templates are required should reflect the modularity of the force (e.g., the level at which forces can be mixed, matched, or tasked).

Clients are owned by entities. It is intended that clients correspond to specific individuals, systems, applications, repositories, or platforms. For example, an F-15 client may be owned by a fighter squadron entity. A client will interface directly with the JBI on behalf of its owner. Unlike entities, clients may not decompose into subcomponents. The entity that owns a client must be registered before the client can connect to the JBI platform. Entities at any level may own a distinct set of clients. The entity client relationship is illustrated in Figure 2.

A pass is an electronic description of a client that enables it to interface with the JBI. The pass defines what a client may do when connected to the JBI. This is primarily expressed in terms of authorized client publications and subscriptions. The information in the pass must be consistent with the force template of the entity that owns the client. The differences between force templates and passes are summarized in Table 1.

\begin{tabular}{|c|c|c|}
\hline \multicolumn{3}{|c|}{ Table 1 - Comparison of Force Template and Passes } \\
\hline & Force Template & Pass \\
\hline Purpose & Register entities with JBI & Register clients with JBI \\
\hline $\begin{array}{l}\text { Activation } \\
\text { Prerequisite }\end{array}$ & $\begin{array}{l}\text { Approval of Joint Force Commander } \\
\text { (JFC) or parent entity }\end{array}$ & $\begin{array}{l}\text { Registration of owner entity's force } \\
\text { template with the JBI }\end{array}$ \\
\hline JBI Interface & Force template controller & Client adapter \\
\hline Content Characteristics & $\begin{array}{l}\text { Distributed, hierarchical, } \\
\text { decomposable }\end{array}$ & Consolidated, cannot be decomposed \\
\hline Minimum Contents & $\begin{array}{ll}- & \text { Entity info requirements } \\
- & \text { Entity info products } \\
- & \text { Entity level constraints } \\
- & \text { Passes for clients owned by the } \\
& \text { entity }\end{array}$ & $\begin{array}{ll}- & \text { Info object advertisements } \\
- & \text { Subscription requests } \\
- & \text { Client level constraints }\end{array}$ \\
\hline
\end{tabular}


Force template contents. There is a wide spectrum of information that the force template could potentially provide the JBI. Some items are essential for the operation of the JBI; others are extensions of the capabilities outlined in the SAB report. As a result, three separate categories are used to characterize force template content; these are: necessary, desired, and speculative (also see Figure 3).

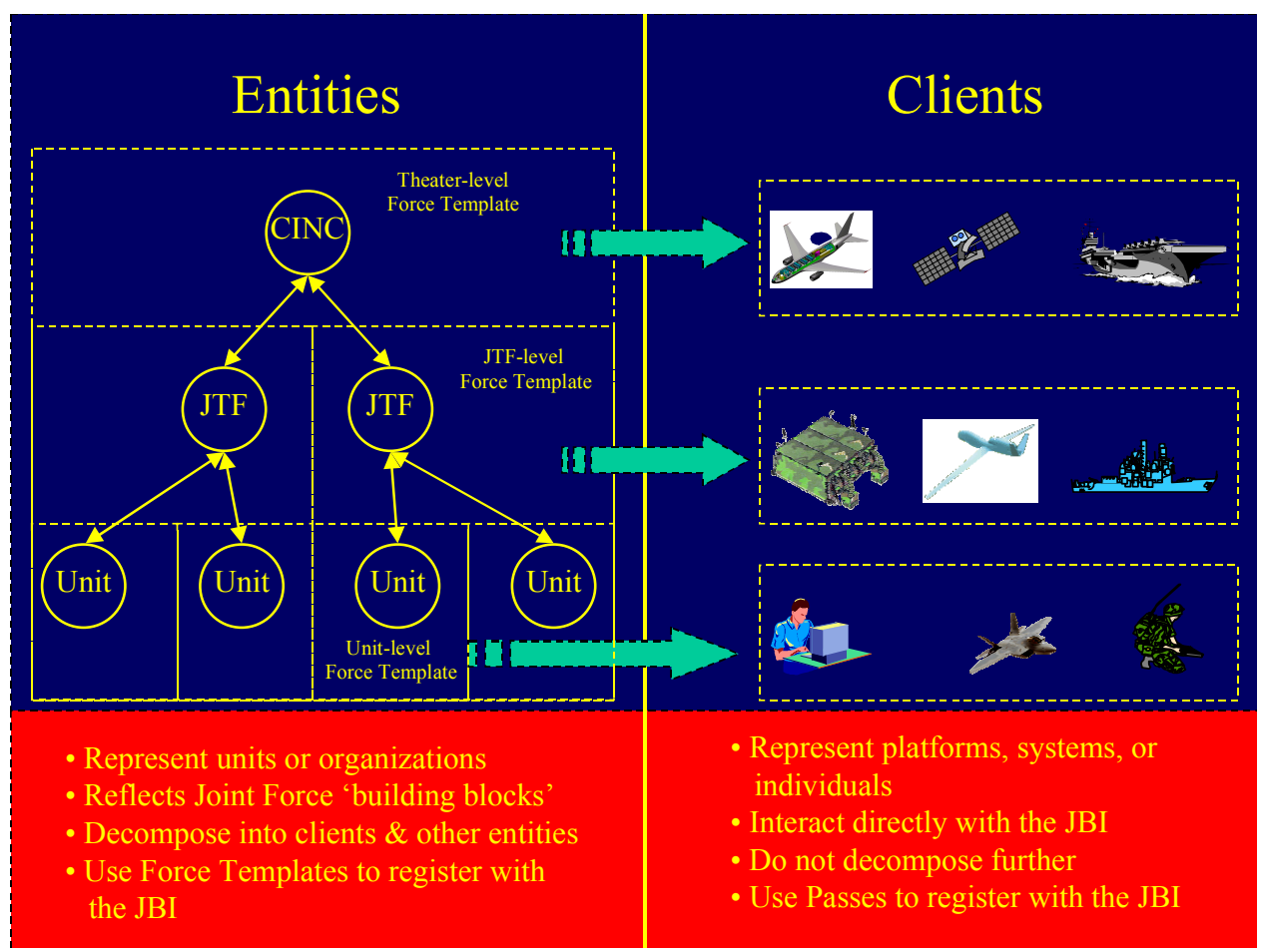

Figure 2 - Entity/Client Relationship

\section{Necessary Contents:}

Information needed by the entity. This refers to information that the entity says it needs to function within the theater. Information can be requested in terms of categorical requirements (expressed as a metadata query) or in terms of specific information object types (predefined subscription requests).

Information provided by the entity. This refers to information that the entity says it can provide within the theater. These will likewise be expressed using metadata descriptions or in terms of specific information object types (advertisements).

The constraints associated with the above. In many cases, information provided or requested will have constraints associated with it. Examples of subscriber constraints include desired quality of service, pedigree, preferred sources, and required delivery windows. Examples of publisher constraints include: anticipated publication times and rates, and dissemination constrains. These constraints may also be expressed in terms of rules about information object content. In this case, publisher advertisements may also include information on publisher capabilities (such as filtering and query capabilities). The JBI platform will use these constraints to broker information requirements against available information products

Security Information. This is a broad and evolving category. The force template could provide a number of security related items to the JBI. This may include:

- The identity and security credentials for individuals occupying key unit positions.

- Public keys for specific clients (individuals, platforms, or systems). 
- Dissemination limitations on published information.

\section{Desired Contents:}

Information Pedigree. This refers to indicators of the quality, reliability, and integrity of entity publications. As such, pedigree ratings may be provided in part by the entity (self-assessment) and in part by the JBI (based on previous history or consumer experience).

Mapping of Specific Personnel to Operational Roles. Force templates for similar units will have a high degree of commonality that extends to positions within the unit. The force templates will communicate to the JBI which personnel are authorized to function in those positions. This mapping could enable the JBI Info Management Staff (IMS) to issue the proper security certificates for those individuals.

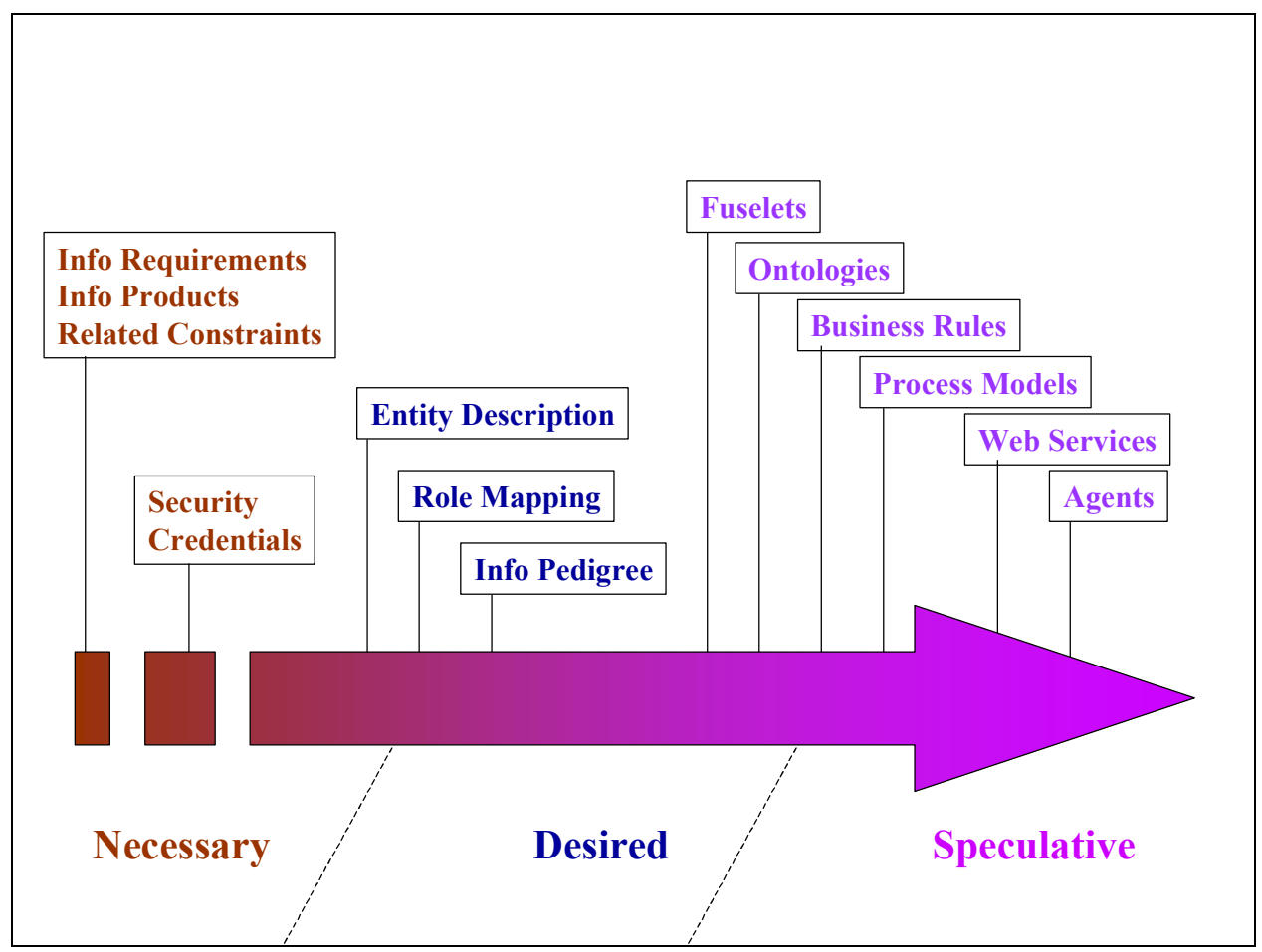

Figure 3 - Force Template Content

Entity Description. This will describe the characteristics of the entity interfacing with the JBI. Ideally, this will take the form of a "resource map" (similar to an active directory) that describes all entity components (e.g., devices, clients, data sources, and people) visible to the JBI. It also includes the child entities that compose the entity (e.g., squadrons within a wing). Each item on the map will list the characteristics of the particular resources. Examples of some unit characteristics include: mission description, unit organizational structure, location, capability description, resource maps, and pointers to associated force templates.

\section{Speculative Contents:}

Ontologies and Ontology Mappings. The more diverse the coalition, the greater the importance of shared semantics. For coalition operations to be successful, it is essential that a consistent set of terms be used to facilitate information sharing [1]. As a result, it is desirable to include ontologies specific to 
an entity, system, or related domain. Whenever possible, these ontologies should come with mappings to common ontologies utilized within the JBI.

Fuselets. Fuselets may be associated with either publications or subscriptions. Examples include XSLT, Excel spreadsheets, Active-X components, or Java beans. Ideally, the force template would contain references to fuselets available from the entity. These fuselets should be associated with specific publications within the JBI (but not necessarily by the providing entity).

Process Models, Rules, and Constraints. These items describe how the entity does business in the theater of operations. Ideally, these will be specified in terms of the included ontologies.

Available Services, or Agents. These items describe services provided by the entity for use by other (appropriate) JBI entities. Examples of services might include: computation of look angles for satellites, requests for surveillance of certain areas, and agent services for determining unit personnel location and status.

\section{Entity/Client Interaction Model}

The SAB report painted a general picture of what the JBI should do and what technologies it might leverage. It did not, however, provide guidance on how the JBI should behave. Since there is no official model for interaction with the JBI, we will take a first cut developing one here. The model proposed here (summarized in Figure 4) ensures the following requirements are met:

- The JBI platform has visibility and control over its inputs and outputs.

- Entities maintain control over what their clients are allowed to do within the JBI through the force template infrastructure.

- Dynamic changes to the force template can be made after registration, allowing the flow of information to evolve during the mission. These changes may be initiated by the top down (from the parent entity or the JBI information staff) or from the bottom up (by the client).

- The integrity and consistency of associated force templates and passes are maintained.

The first part of the model deals with the registration of the entity with the JBI. The notional steps in the process are listed below.

1. Locate the appropriate JBI.

2. Entity requests permission to connect to JBI platform.

3. JBI requests force template package from entity.

4. The entity transmits its force template to the JBI platform.

5. JBI processes force template package.

6. JBI tenders response: acceptance, partial acceptance, or rejection.

7. If acceptance is granted, a controller process is elaborated for the force template.

As discussed earlier, the entity must register prior to registration of its clients. Clients will not be allowed to register with the JBI until an acceptance or partial acceptance is tendered. It is assumed that child entities are not required to register before their parents. This feature offers flexibility in extending the JBI in cases such as when individual squadrons deploy to a theater without their parent wing.

The acceptance of the entity's force template triggers the allocation of a Force Template Controller (FTC) within the JBI platform. The FTC is a gatekeeper that ensures clients behave in a manner consistent with the force template. It also controls changes to the force template that may occur during the entity's JBI session. These changes may be initiated from the bottom up (e.g., client wishes to publish a new information object type) or from the top down (e.g., parent of entity or JBI information staff mandates changes to the force template). 
The proposed client interaction model is illustrated above. The steps for registration of individual clients are listed below.

1. The FTC ensures that adapter processes are elaborated for each client associated with the entity's force template.

2. The passes associated with the clients are cleared for activation within the JBI. The individual clients may attempt connection to the JBI.

3. The client registers with the JBI through its associated adapter.

4. The adapter validates the client. It then receives permission to interact with the JBI in accordance with its pass.

5. If the pass is not validated, permission to interact is denied.

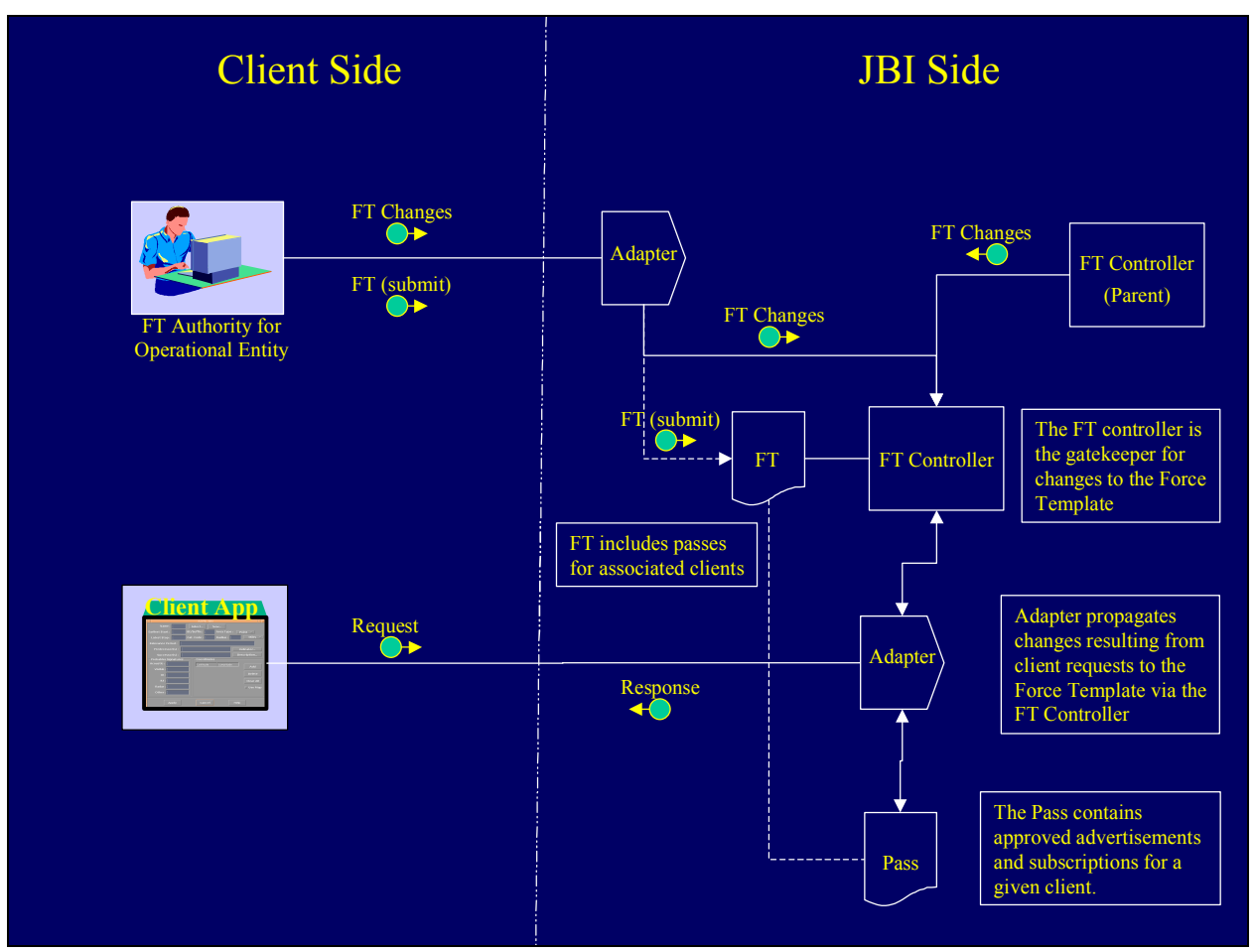

Figure 4: Strawman Force Template Interaction Model

As discussed earlier, the force template contains all passes associated with the entity's clients. The pass contains the approved advertisements and subscriptions for a given client (refer to Table 1). After the entity registers, its passes are maintained by the JBI platform. When the client registers, it submits an encoded reference to the pass that is compared to the version on the JBI side. If they match, the client is given permission to interact with the JBI; otherwise, permission is denied.

Once successfully registered, the client can then initiate JBI operations (e.g., advertise, publish, subscribe, and query) for approved information objects. If the client needs to change its profile, this request is forwarded to the corresponding FTC (through the client's adapter). If the request is consistent with the force template permissions, then an affirmative response is sent back to the client. As a result, the client's adapter on the JBI platform updates the pass. If a negative response is given, however, the request is elevated to the appropriate authorizing authority for further consideration. 
Correspondingly, if changes are directed from above (the legitimate authority within the entity, a parent of the entity, or from the JBI information staff), then those changes are also routed through the FTC. Since these changes are directed (not requested), the force template is automatically updated. This causes the changes to propagate back down to the passes of the affected clients. These changes may result from higher level approval of a client's request that was initially denied by the FTC.

Note that the copy of the force template, and associated passes, updated during the mission is the one maintained by the JBI platform. The entity still retains its copy of the original force template submitted. Because the entity can access (copy) the current force template at any time, it can choose to save versions of the force template as it evolves. If desired, these saved versions can then be used in the future (instead of starting over with the original).

\section{Impact on Coalition C2I Operations}

In this section we discuss how the force template model enhances coalition C2I. For the sake of this exercise, it is assumed that all in-theater coalition possess the credentials and systems necessary to interface with the JBI. Recall that when each coalition member registers with the JBI, their force template will (at a minimum) define what information they need, what they have, and the constraints associated with each.

Although the JBI will be primarily oriented toward military forces, the force template mechanism will provide the flexibility to accommodate relatively ad-hoc coalitions. To be successful, military operations other than war (MOOTW) will require the participation of a wide variety of organizations, including local civil authorities and NGOs [2]. As a result, future C2I systems must be designed with these organizations in mind and provide flexible, appropriate mechanisms for interfacing with them. In cases where these organizations are operating in-theater, they can help provide essential services, such as humanitarian relief, and may (indirectly) serve as important sources of intelligence. In turn, these organizations must be protected without compromising military operations. Successfully integrating these organizations into a common C2I environment will be complicated by the fact they have fundamentally different missions, practices, ontologies, and equipment from the involved military units. While not a total solution, the force template acts as a general-purpose repository for information that describes these aspects of each entity; future C2I applications can draw on these building blocks to overcome these problems.

Regardless of the coalition member's identity, their validated force template will serve as the basis for deciding how their information is utilized, and by whom. Once an entity registers with the JBI, the information products they promise to provide can be brokered according to their specified constraints. This enables each coalition member's information requirements to be intelligently matched with the resources designated as accessible to that member. As part of this process, the JBI will identify the available fuselets that can be used to transform sensitive published information into a form that is releasable to the coalition member. The JBI user will also be able to browse resource directories and identify useful categories of information objects not currently available to him (if those entries are not masked). Once identified, the member can use his force template as the basis for negotiating access to these resources from the publisher.

Although there is no guarantee that all of a coalition member's information requirements will be satisfied by this process, it enables him to leverage the full range of resources (both information and services) available to meet his needs. Given this, the coalition member may be able to satisfy his needs from an ad-hoc collection of available sources, rather than relying on a single source. Thus, in contrast instead of the wholesale denial of information that commonly occurs today, the JBI infrastructure will make it possible for the member to get some subset of what he needs. Within this context, the force template serves as an important enabling mechanism to fashion flexible, information solutions for a diverse set of coalition users.

\section{Conclusion}

If the last decade is any guide, future military operations will be carried out by dynamic, diverse coalitions composed of military, civil, and NGO members. The key to success in these operations will be 
insuring that these entities can quickly exchange both information and service resources within an information-centric C2I infrastructure (infosphere). We have introduced the force template as an enabling mechanism to facilitate this interaction. In this paper, we have taken a first cut at the force template concept by defining what it might contain. We also introduced a model for how it can be used to integrate, and control the interaction of, operational entities (including their children and clients) with the JBI infrastructure. Ultimately, the force template serves as a repository for mission critical information about a battlespace entity; this information includes its identity, what it wants, what it has to offer, and how it intends to operate within the theater. With these items, the infosphere will be able perform contextual brokering of the available resources of each infosphere member. The net result is that infospheres, such as the JBI, can become flexible platforms for the exchange of information and services among coalition partners, insuring (to the extent possible) that the right resource gets to the right member at the right time.

\section{References}

1. Anderson, R.H., and Baer, W. (2000) "Potential Military Applications of Peer-to-Peer Computer Networks", DRR-2670-RC, RAND Corporation, November 2001.

2. Estilow, R.A. (1993) "US Military Force and Operations Other Than War: Necessary Questions to Avoid Strategic Failure”, The Maxwell Papers, No 3, August 1996.

3. United States Air Force Execution Office, "Final Report - Joint Expeditionary Force Experiment 1999", 3 December 1999, pages 44-45.

4. United States Air Force Execution Office, "Alliance Participation Lessons Learned Supplement to: Final Report - Joint Expeditionary Force Experiment 2000".

5. United States Air Force Scientific Advisory Board, "Report on Information Management to Support the Warrior", SAB-TR-98-02, December 2000.

6. United States Air Force Scientific Advisory Board, "Report on Building the Joint Battlespace Infosphere", SAB-TR-99-02, December 1999. 